\title{
Ataque e Caracterização de Danos de Brevicoryne brassicae (L.) (Hemiptera: Aphididae) em Brachiaria decumbens Stapf e Panicum maximum Jacq.
}

\author{
Silma da Silva Camilo, Márcia Vitória Santos ${ }^{1}$, Marcus Alvarenga Soares ${ }^{1}{ }^{\circledR}$ \& Evaldo Martins Pires ${ }^{2}$
}

1. Universidade Federal dos Vales do Jequitinhonha e Mucuri (UFVJM), e-mail: dsilma@yahoo.com.br, marciavito@hotmail.com, marcusasoares@yahoo.com.br (Autor para correspondência ${ }^{\bowtie}$ ). 2. Universidade Federal de Mato Grosso (Campus Sinop-MT), e-mail: evaldo.pires@gmail.com.

\section{EntomoBrasilis 7 (1): 72-74 (2014)}

Resumo. As pastagens cultivadas constituem a base da bovinocultura no Brasil sendo que, os gêneros Brachiaria e Panicum ocupam lugar de destaque. Brachiaria decumbens Stapf possui vantagens como grande adaptabilidade a solos ácidos e de baixa fertilidade, ao clima tropical, além de proporcionar alto rendimento de massa seca. Panicum maximum Jacq. é importante devido ao alto potencial de produção de massa seca por unidade de área, adaptabilidade, qualidade de forragem, facilidade de estabelecimento e aceitabilidade pelos animais. Foi observado o ataque do pulgão Brevicoryne brassicae (L.), comumente encontrado em hortaliças, principalmente nas do gênero Brassica, nestas gramíneas, causando danos como amarelecimento de folhas, depauperamento e formação de fumagina em plantas cultivadas em casa de vegetação. Este inseto poderá se tornar uma praga potencial de $B$. decumbens cv. Basilisk e P. maximum cv. Tanzânia se for confirmada sua adaptação aos novos hospedeiros em campo.

Palavras-chave: Adaptação; Afídeo; Forrageiras.

\section{Attack and Damage Characterization of Brevicoryne brassicae (L.) (Hemiptera: Aphididae) in Brachiaria decumbens Stapf and Panicum maximum Jacq.}

\begin{abstract}
The cultivated pastures are the basis of cattle rearing in Brazil and the Brachiaria and Panicum genera are most important. Brachiaria decumbens Stapf has great advantages such as adaptability to acid soils of low fertility, to the tropical climate and to providing high yield of dry matter. Panicum maximum Jacq. is important because of the high potential production of dry mass per unit area, adaptability, forage quality, ease of establishment and acceptability by animals. It was reported the attack and damage of the aphid Brevicoryne brassicae (L.), commonly found in vegetables, especially in the genus Brassica, in these grasses causing damage such as leaf yellowing, plant depletion and formation of sooty mold in the greenhouse. This insect can become a potential pest of $B$. decumbens cv. Basilisk and P. maximum cv. Tanzânia, if confirmed their adaptation to new hosts in the field.
\end{abstract}

Keywords: Adaptation; Aphid; Forage.

(U) Brasil apresenta cerca de 172 milhões de hectares de pastagens, que correspondem a quase $22 \%$ de seu território (MARTUSCELLO et al. 2012). Aproximadamente $90 \%$ dos nutrientes consumidos pelos ruminantes são originados a partir de plantas forrageiras (EucLides et al. 2010). A criação animal em pasto contribuiu para o sucesso atingido no setor de bovinocultura, pois originou um diferencial qualitativo para a carne brasileira no mercado internacional (Almeida et al. 2011). A predominância de sistemas de produção baseados na utilização dos pastos ocorre, principalmente, devido ao menor custo de produção nestas condições, com menores gastos em mão de obra, combustível, máquinas e com operações envolvidas na alimentação do gado (SANTos et al. 2013).

Brachiaria é um gênero de gramíneas forrageiras de origem africana que tem ganhado importância considerável em regiões tropicais de todo o mundo (ArRoYave et al. 2011). No Brasil, capins deste gênero são os mais cultivados, pois possuem vantagens como grande adaptabilidade a solos ácidos e de baixa fertilidade, ao clima tropical, além de proporcionar alto rendimento de massa seca (DA Silva et al. 2012). Uma destas espécies, a Brachiaria decumbens Stapf (Poaceae) foi introduzida no país na década de 70, coincidindo com a abertura da região de Cerrado (BODDEY et al. 2004). Geralmente, a B. decumbens tem sido conduzida, predominantemente, sob pastejo contínuo, que é um método de maior facilidade operacional (SANTos et al. 2013).

O gênero Panicum é amplo, incluindo algumas espécies economicamente importantes, tais como Panicum maximum Jacq. (Poaceae) (Pessim et al. 2010). Esta gramínea também é proveniente da África, sendo que as primeiras sementes chegaram ao Brasil em camas de palha nos navios negreiros (BoDdey et al. 2004). O P. maximum é importante devido ao alto potencial de produção de massa seca por unidade de área, adaptabilidade, qualidade de forragem, facilidade de estabelecimento e aceitabilidade pelos animais (Tomaz et al. 2010; Gomes et al. 2011).

As plantas forrageiras são cultivadas em extensas áreas na forma de monocultivos no Brasil. Este sistema de cultivo tem como principal característica a redução da biodiversidade do agroecossistema, o que pode favorecer o surgimento de pragas e doenças. Existem vários registros de problemas causados pela monocultura de espécies forrageiras, entre estes a incidência de cigarrinhas das pastagens em $B$. decumbens, de fungos foliares em P. maximum cv. Tanzânia e da morte súbita em Brachiaria

Agências de Financiamento: CNPq, CAPES e FAPEMIG 
- brizantha (Hochst. ex A. Rich.) Stapf. cv. Marandu, o que contribui para a degradação destas pastagens (MARTUSCELLO et al. 2012).

Este trabalho foi proposto com o objetivo de relatar a infestação do pulgão $B$. brassicae em B. decumbens cv. Basilisk e $P$. maximum cv. Tanzânia e caracterizar seus danos nestas espécies vegetais.

A infestação foi detectada em plantas cultivadas em casa de vegetação no setor de Forragicultura do Campus Juscelino Kubitscheck da Universidade Federal dos Vales do Jequitinhonha e Mucuri (UFVJM) em Diamantina (MG), Brasil. O Campus está localizado entre as coordenadas: Latitude $18^{\circ} 15^{\prime}$ Sul, Longitude $43^{\circ} 36^{\prime}$ Oeste e altitude média de $1394 \mathrm{~m}$. O tipo climático da região, de acordo com a classificação de Köepen, é o Cwa (Clima subtropical úmido).

Em junho de 2013 foi observado o ataque intenso de um pulgão em plantas de $B$. decumbens $\mathrm{cv}$. Basilisk e $P$. maximum cv. Tanzânia cultivadas em vasos de polietileno (5 L) (Figuras $1 \mathrm{~A}$, B). Alguns insetos foram coletados das plantas, acondicionados em tubos de vidro contendo álcool etílico 70\% e levados para o laboratório de Entomologia Agrícola da UFVJM. Estes pulgões foram observados em microscópio óptico, com aumento de quatro vezes e identificados como Brevicoryne brassicae (L.) (Hemiptera: Aphididae).

As gramíneas foram atacadas pelo pulgão logo após a poda, quando estavam na fase de rebrotação. Os perfilhos mais novos foram os que apresentaram maior infestação do inseto. Os danos observados foram o amarelecimento de folhas, depauperamento de plantas e formação de fumagina.

O pulgão $B$. brassicae é nativo do continente europeu, mas é comumente encontrado na Ásia, Austrália e Américas (AHMAD \& AKhtar 2013). Este inseto é praga do gênero Brassica (Brassicaceae), que inclui couve, couve-flor, repolho, nabo, brócolis e couve de Bruxelas (Leite et al. 2011; AнMAD \& AKHTAR 2013; Melo et al. 2013) e de batata-doce (Convolvulaceae) (CASTro et al. 2013).

A praga alojava-se em aglomerados e alimentava-se, principalmente, na parte abaxial das laminas foliares mais jovens. Em condições desfavoráveis ao desenvolvimento das plantas, os danos provocados pela alimentação de grande número de pulgões podem ocasionar a morte de plântulas (AHMAD \& AKHTAR
2013). A sucção contínua de seiva provoca clorose e enrugamento foliar, além de introduzir toxinas no sistema vascular da planta. Produzem excremento açucarado que provoca o crescimento de fungos conhecidos como fumagina na superfície das folhas, reduzindo a área fotossintética (LeITE et al. 2011).

Pulgões caracterizam-se por apresentar reprodução assexuada e sexuada. O tempo de desenvolvimento curto pode resultar em 15 a 20 gerações por ano, dependendo das condições climáticas (YusuF \& Collins 1997). Assim, esses insetos atingem, facilmente, altas densidades, o que compromete a qualidade e produtividade das culturas que atacam (Girousse et al. 2003; AhMAD \& Aslam 2005). Seu corpo possui coloração verde acinzentada e apresenta produção de cera branca (AHMAD \& AKHTAR 2013), sendo que, quando adulto a secreção desta substância é tão abundante que cobre totalmente o tegumento (PEREIRA \& LOMÔNACO 2001).

O pulgão B. brassicae se apresentou como uma praga potencial em $B$. decumbens cv. Basilisk e P. maximum cv. Tanzânia, em plantas cultivadas em vasos, em Diamantina - MG, podendo causar danos severos e depauperamento de plantas, especialmente na fase de rebrotação, quando as plantas estão se recuperando após corte ou pastejo por animais. Quando um inseto se adapta em uma nova planta hospedeira, implica-se na aceitação da mesma pelo indivíduo, que pode tornar-se capaz de metabolizar substâncias e nutrientes em concentrações diferentes daquelas de costume (Tosh et al. 2003). Neste caso, a presença de pastagens em grandes áreas de monoculturas no estado de Minas Gerais e no Brasil podem potencializar o ataque destes herbívoros, como já observado para outras culturas (Pires et al. 2011; Menezes et al. 2012; SouZA et al. 2012).

Este é o primeiro registro do ataque de $B$. brassicae em $B$. decumbens cv. Basilisk e $P$. maximum cv. Tanzânia, em casa de vegetação. Devido às grandes áreas de pastagens em monocultivo com essas espécies forrageiras no país, o $B$. brassicae pode se tornar uma praga de grande importância à pecuária brasileira. Todavia, mais estudos são necessários, incluindo trabalhos de campo, objetivando avaliar a relação entre $B$. brassicae e seus novos hospedeiros. Assim, se for confirmada como uma praga em potencial em pastagens, a mesma deve ser monitorada e manejada a partir de práticas de Manejo Integrado de Pragas (MIP), buscando prevenir e, ou, minimizar os danos econômicos nessas culturas.
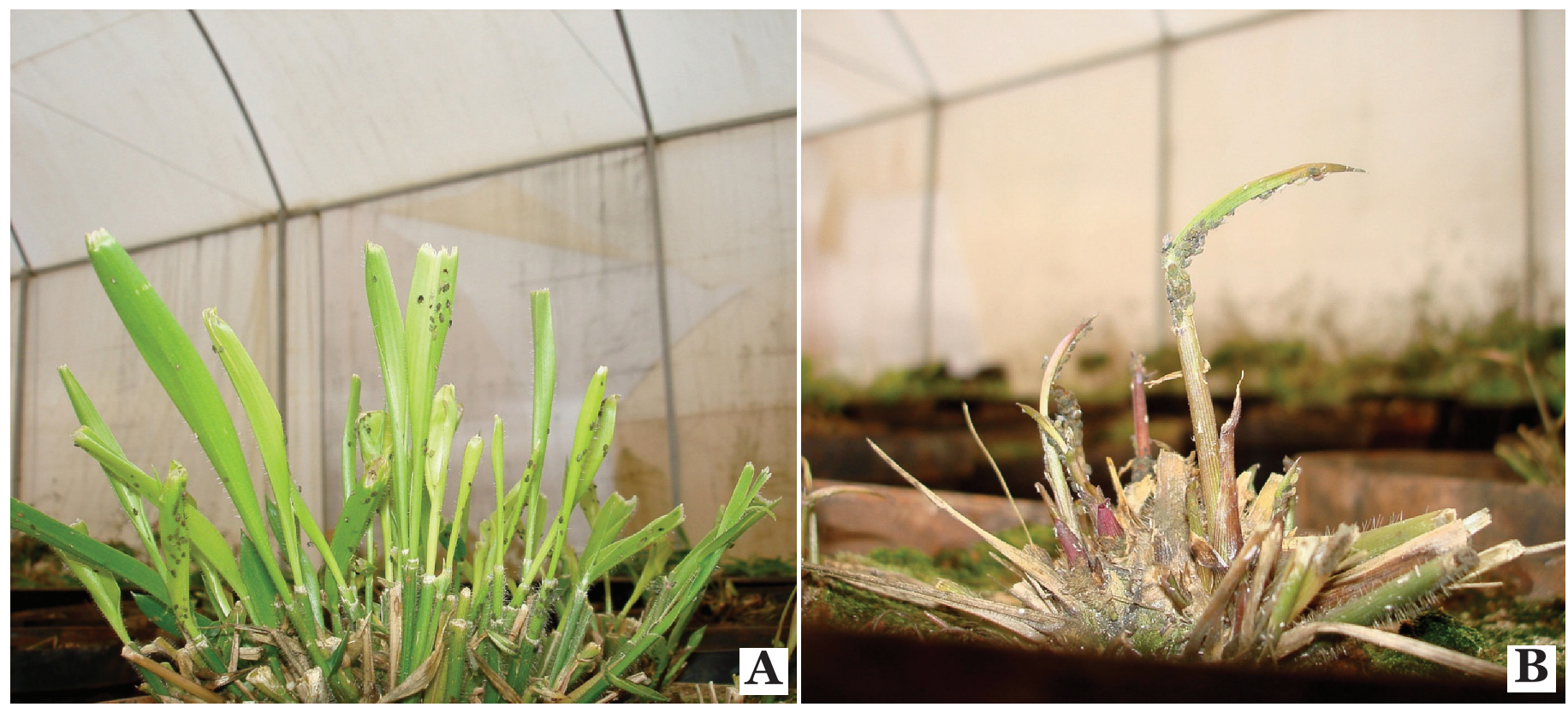

Figura 1. Infestação de Brevicoryne brassicae (Hemiptera: Aphididae) em Brachiaria decumbens (A) e Panicum maximum (B). Diamantina, Minas Gerais, Brasil, 2013. Fotos: Marcus Alvarenga Soares. 


\section{AGRADECIMENTOS}

Às agências brasileiras Conselho Nacional de Desenvolvimento CientíficoeTecnológico-CNPq, CoordenaçãodeAperfeiçoamento de Pessoal de Nível Superior - CAPES e Fundação de Amparo a Pesquisa do Estado de Minas Gerais - FAPEMIG, pelas bolsas e auxílios concedidos.

\section{REFERÊNCIAS}

Ahmad, M. \& S. Akhtar, 2013. Development of insecticide resistance in field populations of Brevicoryne brassicae (Hemiptera: Aphididae) in Pakistan. Journal of Economic Entomology, 2: 954-958.

Ahmad, M. \& M. Aslam, 2005. Resistance of cabbage aphid, Brevicoryne brassicae (Linnaeus) to endosulfan, organophosphates and synthetic pyrethroids. Pakistan Journal of Zoology, 37: 293-295.

Almeida, M.C.C., L. Chiari, L. Jank \& C.B. Valle, 2011. Diversidade genética molecular entre cultivares e híbridos de Brachiaria spp. e Panicum maximum. Ciência Rural, 41: 1998-2003.

Arroyave, C., J. Barceló, C. Poschenrieder \& R. Tolrà. 2011. Aluminium-induced changes in root epidermal cell patterning, a distinctive feature of hyperresistance to $\mathrm{Al}$ in Brachiaria decumbens. Journal of Inorganic Biochemistry, 105: 1477-1483.

Boddey, R.M., R. Macedo, R.M. Tarré, E. Ferreira, O.C. Oliveira, C.P. Rezende, R.B. Cantarutti, J.M. Pereira, B.J.R. Alves \& S. Urquiaga. 2004. Nitrogen cycling in Brachiaria pastures: the key to understanding the process of pasture decline. Agriculture, Ecosystems and Environment, 103: 389-403.

Castro, B.M.C., M.A. Soares, V.C. Andrade-Júnior \& E.M. Pires, 2013. Batata-doce (Ipomoea batatas (L.) Lam.): Um novo hospedeiro para Brevicoryne brassicae (L.) e Myzus persicae (Sulzer) (Hemiptera: Aphididae). Comunicata Scientiae, 4: 220-223.

Da Silva, T.C., A.F. Perazzo, C.H.O. Macedo, E.D. Batista, R.M.A. Pinho, H.F.C. Bezerra \& E.M. Santos, 2012. Morfogênese e estrutura de Brachiaria decumbens em resposta ao corte e adubação nitrogenada. Archivos de Zootecnia, 61: 91-102.

Euclides, V.P.B., C.B. Valle, M.C.M. Macedo, R.G. Almeida, D.B. Montagner, R.A. Barbosa, 2010. Brazilian scientific progress in pasture research during the first decade of XXI century. Revista Brasileira de Zootecnia, 5: 151-168.

Girousse, C., M. Faucher, C. Kleinpeter \& J.L. Bonnemain, 2003. Dissection of the effects of the aphid Acyrthosiphon pisum feeding on assimilate partitioning in Medicago sativa. New Phytologist, 157: 83-92.

Gomes, R.A., B. Lempp, L. Jank, G.C. Carpejani \& M.G. Morais, 2011. Características anatômicas e morfofisiológicas de lâminas foliares de genótipos de Panicum maximum. Pesquisa Agropecuária Brasileira, 46: 205-211.

Leite, G.L.D., M. Picanço, J.C. Zanuncio, M.D. Moreira \& G.N. Jham, 2011. Hosting capacity of horticultural plants for insect pests in Brazil. Chilean Journal of Agricultural Research, 3: 383-289.

Martuscello, J.A., T.G.S. Braz, L. Jank, D.N.F.V. Cunha \& D.M. Fonseca, 2012. Genetic diversity based on morphological data in Panicum maximum hybrids. Revista Brasileira de Zootecnia, 41: 1975-1982.

Melo, B.S.C., E. Bleicher, C.H.C.M. Bertini \& J.F. Silva, 2013. Divergência genética entre cultivares comerciais de repolho quanto à preferência do pulgão-da-couve. Pesquisa Agropecuária Brasileira, 48: 459-465.

Menezes, C.W.G., M.A. Soares, S.L. de Assis Júnior, A.J. Fonseca, E.M. Pires \& J.B. dos Santos, 2012. Novos insetos sugadores (Hemiptera) atacando Eucalyptus cloeziana (Myrtaceae) em Minas Gerais, Brasil. EntomoBrasilis, 5: 246-248.

Pereira, C.D. \& C. Lomônaco, 2001. Plasticidade fisiológica e comportamental de Brevicoryne brassicae (L.) (Hemiptera: Aphididae) em duas variedades de Brassica oleraceae L. Neotropical Entomology, 30: 29-35.

Pessim, C., M.S. Pagliarini, L. Jank, A.M.S. Kaneshima \& A.B.M Bonato, 2010. Meiotic behavior in Panicum maximum Jacq. (Poaceae: Panicoideae: Paniceae): hybrids and their genitors. Acta Scientiarum. Agronomy, 32: 417-422.

Pires, E.M., S.M. Bonaldo, J.A.M. Ferreira, M.A. Soares \& S. Candan, 2011. New record of Leptoglossus zonatus (Dallas) (Heteroptera: Coreidae) attacking starfruit (Averrhoa carambola L.) in Sinop, Mato Grosso, Brazil. EntomoBrasilis, 4: 33-35.

Santos, M.E.R., D.M. Fonseca \& V.M. Gomes, 2013. Forage accumulation in brachiaria grass under continuous grazing with single or variable height during the seasons of the year. Revista Brasileira de Zootecnia, 42: 312-318.

Souza, G.K., T.G. Pikart, F.C. Pikart \& J.C. Zanuncio, 2012. Registro de Oncideres saga (Coleoptera: Cerambycidae) em Peltophorum dubium (Leguminosae) no Município de Trombudo Central, Santa Catarina, Brasil. EntomoBrasilis, 5: 75-77.

Tomaz, C.A., C.C. Martins, L.R. Carvalho \& J. Nakagawa, 2010. Duração do teste de germinação do capim-tanzânia. Revista Brasileira de Sementes, 32: 80-87.

Tosh,C.R.,G.Powell,N.D.Holmes\&J.Hardie, 2003. Reproductive response of generalist and specialist aphid morphs with the same genotype to plant secondary compounds and amino acids. Journal of Insect Physiology, 49: 1173-1182.

Yusuf, S. \& G. Collins, 1997. Effect of soil sulphur levels on feeding preference of Brevicoryne brassicae on brussels sprouts. Journal of Chemical Ecology, 24: 417-424.

\section{Recebido em: 10/09/2013}

Aceito em: 21/o1/2014

\section{Como citar este artigo:}

Camilo, S.S., M.V. Santos, M.A. Soares \& E.M. Pires, 2014. Ataque e Caracterização de Danos de Brevicoryne brassicae (L.) (Hemiptera: Aphididae) em Brachiaria decumbens Stapf. e Panicum maximum Jacq.. EntomoBrasilis, 7 (1): 72-74.

Acessível em: doi:10.12741/ebrasilis.v7i1.388
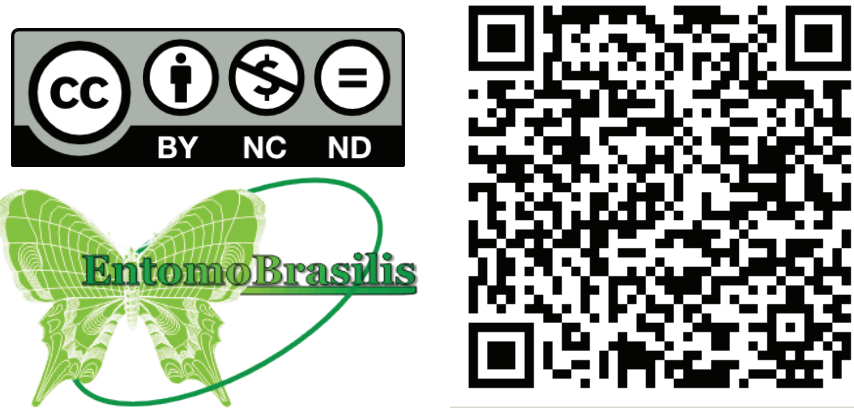